Stewart and Treves described the syndrome of post mastectomy lymphangiosarcoma [1]. A similar clinical entity, forearm CM of breast carcinoma, has been reported $[1,2,3]$.

$\mathrm{CM}$ of breast carcinoma in extremities is very rare, even though the overall incidence is approximately $25 \%$. The commonest presentation is multiple none ulcerated nodules and rarely telangiectatic, erysepeloid, 'en cuirasse' carcinomas, alopecia neoplastica and zosteriform type [3, 4]. We recommend a high degree of suspicion for metastatic lesions in patients presenting with ipsilateral, upper limb cutaneous lesions following breast cancer surgery, although lymphangiosarcoma is commoner.

\section{References}

1. Stewart FW, Treves N. Lymphangiosarcoma in postmastectomy lymphoedema. A report of six cases in elephantiasis chirurgica. Cancer 1948; 1: 64-81.

2. Schafler K, McKenzie CG, Salm R. Postmastectomy lymphangiosarcoma: a reappraisal of the concept - a critical review and report of an illustrative case. Histopathology 1979; 3: 131-52.

3. Frederick PF, Peter MM, Galen P. Extensive cutaneous metastatic breast carcinoma of the hand and upper extremity: a case report. Journal of Hand Surgery 2007; 32: 252-5.

4. Lookingbill DP, Spangler N, Sexton FM. Skin involvement as the presenting sign of internal carcinoma. A retrospective study of 7316 cancer patients. Journal of American Academy of Dermatology 1990; 22: 19-26.

\title{
An unusual presentation of a functioning solitary ectopic thyroid
}

\author{
J Thomas, G A Ghate, P N Shah
}

Ceylon Medical Journal 2014; 59: 69-70

A 14-year old female presented with a swelling in the left submandibular area, which had been present since birth. She had no pain associated with the swelling. On examination a smooth, firm non-tender, non-fluctuant swelling $3 \mathrm{~cm}$ x $2 \mathrm{~cm}$ was detected in the left submandibular region (Figure 1). The swelling was non pulsatile, and did not move with deglutition or protrusion of the tongue. It was non-fluctuant and transillumination test was negative. She had no other swelling in the neck nor was there any cognizant cervical lymphadenopathy. There were no systemic symptoms suggestive of any metabolic disorder. Fine needle aspiration cytology of the swelling revealed abundant thick and thin colloid, thyroid acinar follicles and scattered foamy macrophages. Ultrasonography revealed an ovoid hypo-echoic nodule, with regular contours, measuring $3.4 \times 2.2 \mathrm{~cm}$ in the left submandibular region and the thyroid gland was not visualised at its normal site. Scintigraphy with iodine-131, showed uptake of radiopharmaceuticals in the left submandibular region and no uptake in the normal anatomical site of the thyroid gland (Figure 2) [1]. Thyroid function tests conducted simultaneously revealed that she was euthyroid. In view of the fact that she was euthyroid and since the fine needle aspiration cytology revealed normal functioning thyroid tissue, surgery has been withheld and the patient is undergoing regular follow-up.

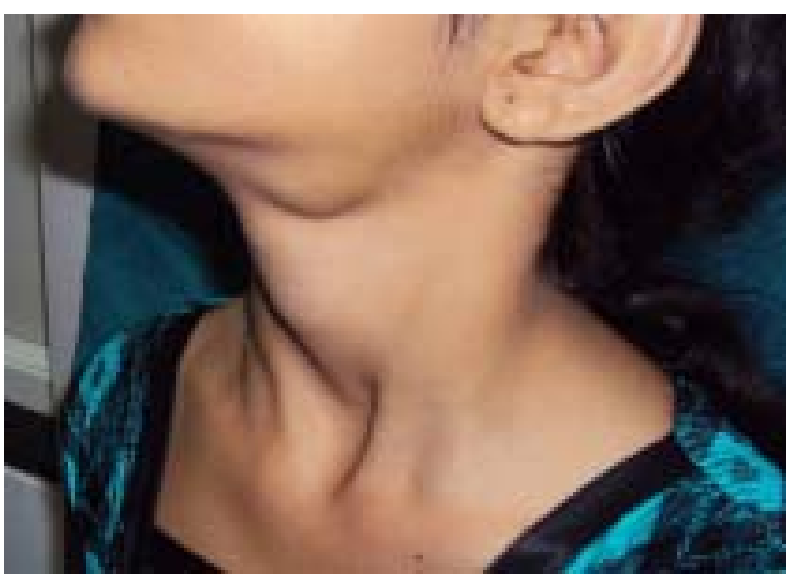

Figure 1.

Department of ENT and HNS, Padmashree Dr. D. Y. Patil Medical College, Pune, India.

Correspondence: JT, e-mail: <jaytees66@yahoo.com>. Received 7 November 2013 and revised version accepted 18 January 2014. Competing interests: none declared. 


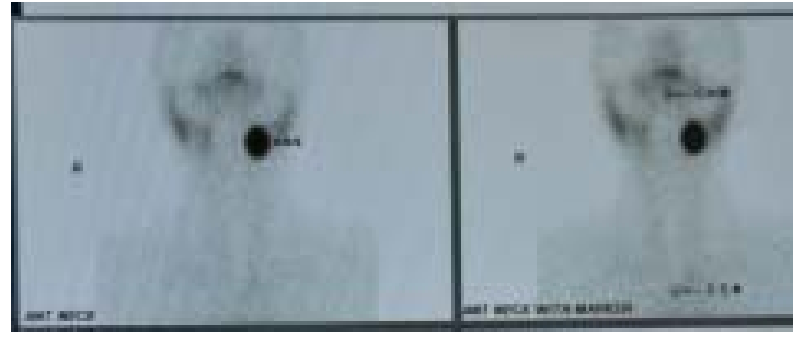

Figure 2.

The occurrence of ectopic thyroid tissue is a relatively rare developmental abnormality which is consequent to aberrant embryogenesis of the primordial thyroid gland during its passage from the floor of the primitive foregut to its final pre-tracheal position [1,2]. Its occurrence is around one per one hundred thousand to three hundred thousand of human population. In clinical practice the most frequent location of ectopic thyroid tissue is at the region of the foramen cecum, accounting for about $90 \%$ of the reported cases [3].

\section{References}

1. Noussios G, Anagnostis P, Goulis DG, et al. Ectopic thyroid tissue: anatomical, clinical, and surgical implications of a rare entity. European Journal of Endocrinology 2011; 165: 375-82.

2. Babazade F, Mortazavi H, Jalalian H, et al. Thyroid tissue as a submandibular mass: a case report. Journal of Oral Science 2009; 51: 655-7.

3. Weber AL, Randolph G, Aksoy FG. The thyroid and parathyroid glands. CT and MR imaging and correlation with pathology and clinical findings. Radiologic Clinics of North America 2000; 38: 1105-29.

\title{
A case of Hymenolepis diminuta (rat tape worm) infestation in a child
}

\author{
V P Sinhabahuํㅜ, T M R Perera ${ }^{1}$, S Samarasinghe $^{2}$
}

Ceylon Medical Journal 2014; 59:70-71

A five-year old boy presented with fever, watery stools and a febrile convulsion on day one of the illness. He has had on and off abdominal pain for 5 months. No urinary symptoms nor blood stained stools noted. He has had a febrile convulsion at 9 months. Growth and development were appropriate for the age. The immunisation was uptodate. Examination was unremarkable. Microscopic examination of faeces showed 10-12 pus cells and 15-16 red cells per high power field and eggs of Hymenolepis diminuta (Figure 1). Stool culture did not yield any significant pathological organism. Stool virology could not be performed. Child was treated with praziquantal $10 \mathrm{mg} / \mathrm{kg}$ as a single dose. A microenema was administered 2 hours later and a 24 hour stool collection was done to check the excretion of the worms which yielded numerous worm segments (Figure 2). A repeat stool examination was done one month later which did not show evidence of $H$. diminuta infestation.

The natural reservoir and definitive host of H.diminuta are rodents. Coprophilic arthropods such as flea and beetle species act as intermediate hosts [1]. Humans act as definitive hosts only when they ingest cysticercoid larvae infected arthropods with food and fomites. This is the reason why $H$. diminuta infestation is uncommon in humans [1]. Only a limited number of cases have been reported globally [1]. H. diminuta is known to cause abdominal pain and loose stools in children [3]. $H$. diminuta infestation has not been reported to cause seizures before. Praziquantel and niclosamide can be used for treatment of $H$. diminuta infestations.

${ }^{1}$ University Paediatric Unit, Colombo South Teaching Hospital, ${ }^{2}$ Medical Research Institute, Colombo, Sri Lanka. Correspondence: VPS, e-mail: <Sinbad.Ik@gmail.com>. Received 30 November 2013 and revised version accepted 29 March 2014. Competing interests: none declared. 